\title{
The chain recurrent set, attractors, and explosions
}

\author{
LOUIS BLOCK AND JOHN E. FRANKE \\ Department of Mathematics, University of Florida, Gainesville, Florida 32611, USA; \\ Department of Mathematics, North Carolina State University, Raleigh, \\ North Carolina 27695, USA
}

(Received 5 November 1984)

\section{Dedicated to the memory of Charles Conley}

\begin{abstract}
Charles Conley has shown that for a flow on a compact metric space, a point $x$ is chain recurrent if and only if any attractor which contains the $\omega$-limit set of $x$ also contains $x$. In this paper we show that the same statement holds for a continuous map of a compact metric space to itself, and additional equivalent conditions can be given. A stronger result is obtained if the space is locally connected. It follows, as a special case, that if a map of the circle to itself has no periodic points then every point is chain recurrent. Also, for any homeomorphism of the circle to itself, the chain recurrent set is either the set of periodic points or the entire circle. Finally, we use the equivalent conditions mentioned above to show that for any continuous map $f$ of a compact space to itself, if the non-wandering set equals the chain recurrent set then $f$ does not permit $\Omega$-explosions. The converse holds on manifolds.
\end{abstract}

\section{Introduction}

This paper is concerned with properties of chain recurrent points and the chain recurrent set for continuous maps. Throughout the paper, we let $X$ denote a compact metric space (with metric $d$ ), $f$ denote a continuous map from $X$ to itself, and $R(f)$ denote the chain recurrent set (defined in $\$ 2$ ).

Our first result (proved in $\$ 2$ ) gives some necessary and sufficient conditions for a point $x \in X$ to be chain recurrent. Before stating this result we recall a few definitions. If $Y$ is a subset of $X$ we set

$$
\omega(Y)=\bigcap_{n=0}^{\infty}\left(\overline{\bigcup_{k \geq n} f^{k}(Y)}\right) .
$$

If $y \in X$, the $\omega$ limit set of $y$ is $\omega(y)=\omega(\{y\})$. Following Conley, [4], we say a subset $K$ of $X$ is an attractor if there is some open set $Y$ containing $K$ with $K=\omega(Y)$.

THEOREM A. Let $x \in X$. The following are equivalent.

(1) $x \notin R(f)$.

(2) There is an open set $U$ with $x \notin \bar{U}, f(x) \in U$, and $f(\bar{U}) \subset U$.

(3) There is an open set $U$ with $x \notin U, f(x) \in U$, and $f(\bar{U}) \subset U$. 
(4) There is an open set $U$ with $x \notin U, f(\bar{U}) \subset U$, and $f^{k}(x) \in U$ for some positive integer $k$.

(5) There is an attractor $K$ with $x \notin K$ and $\omega(x) \subset K$.

We remark that in the case of flows, the equivalence of (1) and (5) was shown by Conley (see [4, p. 37]). Similar ideas were also used by Bowen, [3], and Dowker and Friedlander, [7], in connection with abstract $\omega$ limit sets. In proving theorem A we obtain the following characterization of attractors for maps, which relates Conley's definition of attractor to that of other authors (see [8], [15] and [16] for example).

LEMMA 3. Let $K \subset X . K$ is an attractor if and only if there is an open set $U$ containing $K$ with $f(\bar{U}) \subset U$ and $K=\bigcap_{n=0}^{\infty} f^{n}(\bar{U})$.

The implication $(1) \Rightarrow(5)$ of theorem A says that unless every point is chain recurrent there is a proper subset $K$ of $X$ which is an attractor. In general, as an example in $\$ 3$ shows, there need not exist a connected attractor, even for an iterate of $f$. However, if $\boldsymbol{X}$ is locally connnected, we have the following.

Theorem B. Suppose $X$ is locally connected and $x \in X$. The following are equivalent.

(1) $x \notin R(f)$.

(2) There exists a connected open set $U$ and positive integers $n$ and $k$ such that $x \notin U, f^{n}(\bar{U}) \subset U$, and $f^{k n}(x) \in U$.

(3) For some positive integer $n$, there is a connected attractor $K$ of $f^{n}$ such that $x \notin K$ and $\omega\left(x, f^{n}\right) \subset K$ (where $\omega\left(x, f^{n}\right)$ denotes $\omega(x)$ for the map $\left.f^{n}\right)$.

The following corollary is easily obtained from theorem B.

COROLlaRY C. Let $X$ be connected and locally connected. The following are equivalent.

(1) $R(f) \neq X$.

(2) There is a connected, open set $U$ with $U \neq X$ and $f^{n}(\bar{U}) \subset U$ for some positive integer $n$.

(3) For some positive integer $n$, there is a connected attractor $K$ of $f^{n}$ with $K \neq X$.

In the special case where $X$ is the circle either statement (2) or (3) of corollary C immediately implies the existence of a periodic point. Thus we obtain the following.

COROLlARY D. If $f$ is a continuous map of the circle to itself with no periodic points then every point is chain recurrent.

Corollary D could also be proved by using the result of Auslander and Katznelson given in [1]. Using corollary $D$ we prove the following result for homeomorphisms of the circle.

COROllary E. If $f$ is a homeomorphism of the circle to itself, then $R(f)$ is either the circle or the set of periodic points.

Note that corollaries $\mathrm{D}$ and $\mathrm{E}$ do not hold for the non-wandering set as the example of Denjoy ([6], [9]) shows. 
The final section of the paper $(\$ 4)$ deals with explosions of the chain recurrent set and the non-wandering set. Let $C(X, X)$ denote the space of continuous maps from $X$ to itself with the uniform topology. We say $f$ does not permit $R$-explosions if for every $\varepsilon>0$ there is a neighbourhood $N(f)$ in $C(X, X)$ such that if $g \in N(f)$ then for any $x \in R(g), d(x, R(f))<\varepsilon$. The expression ' $f$ does not permit $\Omega$-explosions' is defined analogously. We emphasize that here we are looking at the $C^{0}$ topology and $C^{0}$ explosions. Using theorem $\mathrm{A}$, we prove:

THEOREM F. If $f$ is any continuous map of a compact metric space $X$ to itself, then $f$ does not permit R-explosions.

As an immediate corollary we have:

Corollary G. If $\Omega(f)=R(f)$ then $f$ does not permit $\Omega$-explosions.

In general the converse of corollary $G$ may be false as an example in $\$ 4$ shows. However, using a form of the closing lemma in the case where $X$ is a manifold we obtain:

THEOREM H. If $X$ is a compact topological manifold then $\Omega(f)=R(f)$ if and only if $f$ does not permit $\Omega$-explosions.

Corollary $\mathrm{G}$ and theorem $\mathrm{H}$ extend similar results proved in other settings (see [10], [11], [13], and [14]). The authors would like to thank David Hart, Zbigniew Nitecki, and R. Clark Robinson for helpful conversations relating to this work.

\section{Proof of theorem A}

We first review some terminology and notation. Recall $X$ denotes a compact metric space and $f$ denotes a continuous map from $X$ to itself. If $x \in X$ and $y \in X$ an $\varepsilon$-chain from $x$ to $y$ is a finite sequence of points $\left\{x_{0}, x_{1}, \ldots, x_{n}\right\}$ of $X$ with $x=x_{0}$, $y=x_{n}$, and $d\left(f\left(x_{i-1}\right), x_{i}\right)<\varepsilon$ for $i=1, \ldots, n$. We let $R_{\varepsilon}(x)$ denote the set of $y \in X$ such that there is an $\varepsilon$-chain from $x$ to $y$. We say $x$ can be chained to $y$ if $y \in R_{\varepsilon}(x)$ for every $\varepsilon>0$, and $x$ is chain recurrent if $x$ can be chained to $x$. The set of all chain recurrent points is denoted by $R(f)$. Finally, we say a subset $Y$ of $X$ is positively chain invariant if for every $y \in Y$ and $x \in X \backslash Y, y$ cannot be chained to $x$.

LEMMA 1. Let $x \in X$ and $\varepsilon>0 . R_{\varepsilon}(x)$ is an open positively chain invariant set with $f\left(\overline{R_{\varepsilon}(x)}\right) \subset R_{\varepsilon}(x)$.

Proof. It follows easily from the definitions that $R_{\varepsilon}(x)$ is open and positively chain invariant. We show that $f\left(\overline{R_{\varepsilon}(x)}\right) \subset R_{\varepsilon}(x)$. Let $y \in \overline{R_{\varepsilon}(x)}$. There is a $\delta>0$ with $\delta<\varepsilon$ such that if $d(y, z)<\delta$ then $d(f(y), f(z))<\varepsilon$. Since $y \in \overline{R_{\varepsilon}(x)}$, there is a $z \in R_{\varepsilon}(x)$ with $d(y, z)<\delta$. Let $\left\{x_{0}, \ldots, x_{n}\right\}$ be an $\varepsilon$-chain from $x$ to $z$. Then $\left\{x_{0}, \ldots, x_{n}, f(y)\right\}$ is an $\varepsilon$-chain from $x$ to $f(y)$, so $f(y) \in R_{\varepsilon}(x)$. Thus $f\left(\overline{R_{\varepsilon}(x)}\right) \subset R_{\varepsilon}(x)$.

We will use the following lemma from [2].

LEMMA 2. Let $S$ be a positively chain invariant subset of $X$. If $x \notin S$ and $f^{k}(x) \in S$ for some positive integer $k$, then $x \notin R(f)$. 
LEMMA 3. Let $K \subset X . K$ is an attractor if and only if there is an open set $U$ containing $K$ with $f(\bar{U}) \subset U$ and $K=\bigcap_{n=0}^{\infty} f^{n}(\bar{U})$.

Proof. Suppose $K$ is an attractor. There is an open set $Y$ containing $K$ with $K=\omega(Y)$. If for each positive integer $i, f^{i}(\bar{Y})$ contained points outside of $Y$ there would be an element of $\omega(Y)$ outside of $Y$, contradicting $\omega(Y) \subset Y$. Thus, for some positive integer $k, f^{k}(\bar{Y}) \subset Y$.

Let $Y_{k-1}$ be an open set containing $f^{k-1}(\bar{Y})$ such that $f\left(\bar{Y}_{k-1}\right) \subset Y$, and let $Y_{k-2}, \ldots, Y_{2}$, and $Y_{1}$ be open sets such that $Y_{i}$ contains $f^{i}(\bar{Y})$ and $f\left(\bar{Y}_{i}\right) \subset Y_{i+1}$ for $i=1, \ldots, k-2$. Let

$$
U=Y_{1} \cup Y_{2} \cup \cdots \cup Y_{k-1} \cup Y
$$

Then $f(\bar{U}) \subset U$ and

$$
K=\omega(Y)=\omega(U)=\bigcap_{n=0}^{\infty} f^{n}(\bar{U}) .
$$

This proves one direction. The other direction is immediate (take $Y=U$ ).

Proof of theorem A. The implications $(2) \Rightarrow(3),(3) \Rightarrow(4)$, and (4) $\Rightarrow(5)$ are immediate. We prove $(1) \Rightarrow(2)$ and $(5) \Rightarrow(1)$.

(1) $\Rightarrow(2)$. For some $\varepsilon>0, x \notin R_{\varepsilon}(x)$. Let $W=R_{\varepsilon}(x)$. By lemma $1, W$ is open and $f(\bar{W}) \subset W$. Also, $x \notin W$ but $f(x) \in W$. We may assume that $x \in \bar{W}$, or else the conclusion follows with $U=W$. There is an open subset $U$ of $X$ with $f(\vec{W}) \subset U \subset$ $\vec{U} \subset W$. Then $x \notin \bar{U}, f(x) \in U$, and $f(\bar{U}) \subset U$.

$(5) \Rightarrow(1)$. By lemma 3 , there is an open set $U$ with $f(\bar{U}) \subset U$ and $K=\bigcap_{n=0}^{\infty} f^{n}(\bar{U})$. Since $x \notin K, x \notin f^{m}(\bar{U})$ for some positive integer $m$. Let $W$ be an open subset of $X$ such that $f^{m}(\bar{U}) \subset W \subset U$ but $x \notin \bar{W}$. Since $f^{m}(\bar{W}) \subset W$ it follows that $\bar{W}$ is positively chain invariant under $f^{m}$. On the other hand, as $\omega(x) \subset K$, we have that $f^{j}(x) \in U$ for some positive integer $j$. Thus, $f^{i}(x) \in \bar{W}$ for all $i \geq j+m$, and in particular, $f^{m k}(x) \in \bar{W}$ for some positive integer $k$. By lemma $2, x \notin R\left(f^{m}\right)$, and since $R\left(f^{m}\right)=$ $R(f)([2],[12]) x \notin R(f)$.

\section{Proof of theorem $\mathrm{B}$ and corollaries}

Proof of theorem B. (1) $\Rightarrow(2)$. By hypothesis, $x \notin R_{\varepsilon}(x)$ for some $\varepsilon>0$. Since $X$ is locally connected, each component of the open set $R_{\varepsilon}(x)$ is open. Thus, the compact set $f\left(\overline{R_{\varepsilon}(x)}\right)$ is covered by a finite number of components of $R_{\varepsilon}(x)$, say $V_{1}, V_{2}, \ldots, V_{k}$. For each $i \in\{1, \ldots, k\}$ there is a unique $j \in\{1,2, \ldots, k\}$ such that $f\left(\bar{V}_{i}\right) \subset V_{j}$. It follows that (2) holds for some $U \in\left\{V_{1}, \ldots, V_{k}\right\}$.

$(2) \Rightarrow(3)$. Let $K=\bigcap_{k=0}^{\infty} f^{k n}(\bar{U})$.

(3) $\Rightarrow$ (1). By $(5) \Rightarrow(1)$ of theorem A, $x \notin R\left(f^{n}\right)=R(f)$.

The proof of corollary $\mathrm{C}$ may easily be obtained by the reader. We remark, however, that this corollary (and theorem B as well) may be false if $X$ is not locally connected. To see this, we construct an example of a homeomorphism $f$ defined on a subset $X$ of the plane. Let $S^{1}$ denote the unit circle on the plane and let $h: S^{1} \rightarrow S^{1}$ denote the Denjoy homeomorphism ([6], [9]). Let $K$ denote the non-wandering set of $h$, and recall that $K$ is homeomorphic to the Cantor set. 
Let $X$ consist of all points $(r, \theta)$ in the plane, in polar coordinates, with $0 \leq r \leq 1$, and $(1, \theta) \in K$. In other words, $X$ consists of the set $K$ together with the line segments drawn from each point of $K$ to the origin. Note that $X$ is compact and connected, but not locally connected. We define a homeomorphism $f: X \rightarrow X$ by $f(r, \theta)=$ $(\sqrt{r}, h(\theta))$. Then $K$ is an attractor for $f$, and by theorem $\mathrm{A}, R(f)$ consists of the set $K$ and the origin (which is a fixed point). Thus, for this example, statement (1) of corollary C holds, but neither (2) nor (3) holds.

COROLlaRY E. If $f$ is a homeomorphism of the circle to itself, then $R(f)$ is either the circle or the set of periodic points.

Proof. Let $S^{1}$ denote the circle. If every point of $S^{1}$ is periodic, then the conclusion is immediate, so we may assume that the set of periodic points of $f$, which we denote by $P(f)$, is a proper subset of $S^{1}$. If $P(f)=\varnothing$, the conclusion follows from corollary $\mathrm{D}$, so we may assume that $P(f) \neq \varnothing$. Since the periodic points of a homeomorphism of $S^{1}$ have at most two different periods (see [9]) and $R(f)=R\left(f^{n}\right)$, we may assume that $P(f)$ consists entirely of fixed points of $f$. Also, again replacing $f$ by $f^{2}$ if necessary, we may assume that $f$ maps each component of $S^{1} \backslash P(f)$ onto itself.

Now, for each component $V$ of $S^{\mathrm{l}} \backslash P(f)$ there is a direction, either clockwise or counterclockwise, such that $f$ moves each point in $V$ in the specified direction. If the directions are the same for all components, then it is easy to verify that $R(f)=S^{1}$. If the directions for two components are different, let $x \in S^{\mathbf{l}} \backslash P(f)$ and let $y$ be in a component with the opposite direction. Let $U$ denote the open interval from $x$ to $y$ which contains $f(x)$ and $f(y)$. Then $x \notin U, f(x) \in U$, and $f(\bar{U}) \subset U$. By theorem A, $x$ is not chain recurrent. Since $x$ was arbitrary, $R(f)=P(f)$.

\section{Explosions}

THEOREM F. If $f$ is any continuous map of a compact metric space $X$ to itself, then $f$ does not permit $R$-explosions.

Proof. Let $\varepsilon>0$ and let $Y=X \backslash N(R(f), \varepsilon)$, where $N(R(f), \varepsilon)=$ $\{x \in X: d(x, R(f))<\varepsilon\}$. Let $y \in Y$. By theorem $A$ there is an open subset $U_{y}$ of $X$ such that $f\left(\bar{U}_{y}\right) \subset U_{y}$ and $y \notin \bar{U}_{y}$ but $f(y) \in U_{y}$. There is an open neighbourhood $O_{y}$ of $y$ such that $f\left(\bar{O}_{y}\right) \subset U_{y}$ and $\bar{O}_{y} \cap \bar{U}_{y}=\varnothing$.

There is a neighbourhood $N_{y}(f)$ of $f$ in $C(X, X)$ such that if $g \in N_{y}(f)$ then $g\left(\bar{U}_{y}\right) \subset U_{y}$ and $g\left(\bar{O}_{y}\right) \subset U_{y}$. By theorem $\mathrm{A}$, this implies that if $g \in N_{y}(f)$ then no point of $O_{y}$ is in $R(\mathrm{~g})$.

The set of $O_{y}$ formed as above for each $y \in Y$ is an open cover of $Y$. Let $\left\{O_{y_{1}}, \ldots, O_{y_{k}}\right\}$ be a finite subcover, and let $N_{y_{1}}(f), \ldots, N_{y_{k}}(f)$ be the corresponding neighbourhoods of $f$ in $C(X, X)$ as above. Let $N(f)=N_{y_{1}}(f) \cap \cdots \cap N_{y_{k}}(f)$. If $g \in N(f)$ then $Y \cap R(g)=\varnothing$.

As an immediate corollary we have:

Corollary G. If $\Omega(f)=R(f)$ then $f$ does not permit $\Omega$-explosions.

Note that, in general, the converse to corollary $\mathrm{G}$ may be false. For example, let $X$ be the subset of the plane given by $X=S^{1} \cup K$ where $S^{1}=\left\{(x, y) \in R^{2}: x^{2}+y^{2}=1\right\}$ 
and $K=\left\{(x, y) \in \mathbb{R}^{2}: x=0\right.$ and $y= \pm(1-(1 / n))$ for some positive integer $\left.n\right\}$. Define $f_{1}$ on $S^{1}$ to be the north pole-south pole homeomorphism (i.e. $(0,1)$ and $(0,-1)$ are fixed points of $f_{1}$ and for all other points $(x, y)$ the second coordinate of $f_{1}(x, y)$ is less than $y)$. Define $f_{2}$ on $K$ by $f_{2}(0,1)=(0,1), f_{2}(0,-1)=(0,-1), f_{2}(0,1-(1 / n))=$ $(0,1-(1 / n+1))$ for each positive integer $n$, and $f_{2}(0,-1+(1 / n))=$ $(0,-1+(1 / n-1))$ for each integer $n \geq 2$. There is a unique map $f$ from $X$ to itself such that $f \mid S^{1}=f_{1}$ and $f \mid K=f_{2}$, and $f$ is a homeomorphism of the compact metric space $X$ to itself. Note that $R(f)=X$ while $\Omega(f)=\{(0,1),(0,-1)\}$. The reader can verify that $f$ does not permit $\Omega$-explosions.

The converse to corollary $G$ is true on topological manifolds. To prove this we need an appropriate closing lemma for our setting. We give a simple proof, similar to the proof for homeomorphisms given in [13] (see also [5]).

LeMma 4. Let $M$ be a compact topological manifold with metric d. Let $N(I)$ be a neighbourhood of the identity map in $C(M, M)$. There is a $\delta>0$ such that if $\left\{\left(p_{1}, q_{1}\right), \ldots,\left(p_{k}, q_{k}\right)\right\}$ is a finite set of points in $M \times M$ satisfying:

(i) for each $i=1, \ldots, k, d\left(p_{i}, q_{i}\right)<\delta$; and

(ii) if $i \neq j$, then $p_{i} \neq p_{j}$ and $q_{i} \neq q_{j}$;

then there is an $h \in N(I)$ with $h\left(p_{i}\right)=q_{i}$ for $i=1, \ldots, k$.

Proof. It is shown in [11] that the conclusion holds if the dimension of $M$ is at least two. In fact, in this case $h$ can be chosen to be a homeomorphism (a diffeomorphism if $M$ is smooth). In the one-dimensional case one easily constructs the map $h$ by sending $p_{i}$ to $q_{i}$ and extending to a piecewise linear map. In this case, of course, $h$ can not always be chosen to be a homeomorphism.

LeMma 5 (Closing Lemma). Let $M$ be a compact topological manifold and $f$ a continuous map of $M$ to itself. If $x \in R(f)$ then for any neighbourhood $N(f)$ in $C(M, M)$ there is a $g \in N(f)$ such that $x$ is a periodic point of $g$.

Proof. Let $N(I)$ be a neighbourhood of the identity map in $C(M, M)$ such that if $h \in N(I)$ then $h \circ f \in N(f)$. Let $\delta>0$ be as in lemma 4 , and let $\left\{x_{0}, x_{1}, \ldots, x_{k}\right\}$ be a $\delta$-chain from $x$ to $x$ with $k$ minimal. It is easy to verify that the set of points $\left\{\left(f\left(x_{0}\right), x_{1}\right), \ldots,\left(f\left(x_{k-1}\right), x_{k}\right)\right\}$ satisfies the hypothesis of lemma 4 , so there is an $h \in N(I)$ such that $h\left(f\left(x_{i}\right)\right)=x_{i+1}$ for $i=0, \ldots, k-1$. If $g=h \circ f$ then $g \in N(f)$ and $x$ is a periodic point of $g$.

Theorem $\mathrm{H}$ now follows immediately from corollary $\mathrm{G}$ and lemma 5.

\section{REFERENCES}

[1] J. Auslander \& Y. Katznelson. Maps of the circle without periodic points. Israel J. Math. 32 (1979), 375-381.

[2] L. Block \& J. Franke. The chain recurrent set for maps of the interval. Proc. Amer. Math. Soc. 87 (1983), 723-727.

[3] R. Bowen. $\omega$-Limit sets for Axiom A diffeomorphisms. J. Differential Equations 18 (1975), 333-339.

[4] C. Conley. Isolated invariant sets and the Morse index. CBMS Regional Conf. Ser. in Math., no. 38, Amer. Math. Soc.: Providence, R.I., 1976. 
[5] E. M. Coven, J. Madden \& Z. Nitecki. A note on generic properties of continuous maps. In Ergodic Theory and Dynamical Systems II. Birkhauser: Boston, 1982.

[6] A. Denjoy. Sur les courbes definies par les equations differentielles a la surface du tore. J. de Math Pures et Appliquees 11 (ser 9) (1932), 333-375.

[7] Y. Dowker \& F. Friedlander. On limit sets in dynamical systems. Proc. London Math. Soc. 4 (1954), 168-176.

[8] S. Newhouse. Lectures on Dynamical Systems. In Dynamical Systems, Progress in Math. Vol. 8. Birkhauser, 1978, pp. 1-114.

[9] Z. Nitecki. Differentiable Dynamics, an Introduction to the Orbit Structure of Diffeomorphisms. M.I.T. Press: Cambridge, Massachusetts, 1971.

[10] Z. Nitecki. Explosions in completely unstable flows; I: preventing explosions. Trans. Amer. Math. Soc. 245 (1978), 43-61.

[11] Z. Nitecki and M. Shub. Filtrations, decompositions, and explosions. Amer. J. Math. 97 (1976), 1029-1047.

[12] K. Sawada. On the iterations of diffeomorphisms without $C^{0}-\Omega$-explosions: an example. Proc. Amer. Math. Soc. 79 (1980), 110-112.

[13] M.Shub. Stabilité-globale des systemes dynamiques. Asterisque 56, Societe Mathematique de France, Paris, 1978.

[14] M. Shub \& S. Smale. Beyond hyperbolicity. Annals of Math. 96 (1972), 578-591.

[15] R. F. Williams. The zeta function of an attractor. In Michigan State meeting on Algebraic Topology. Prindle, Weber \& Smith, pp. 155-161.

[16] R. F. Williams. The structure of attractors. Proc. International Congress Math. 2 (1970), 947-951. 